\title{
Green Synthesis of
}

Novel 5-Arylazo-2-[(2S, 3S, 4R, 5R)-3, 4, 5-Trihydroxy-6-(Hydroxymethyl) Tetrahydro-2H-Pyran-2-Yloxy]-4, 6-Dimethyl 3-Nicotinonitrile

\author{
Magda H. Abdellattif ${ }^{*}$, Mohamed Mohamed Helmy Arief ${ }^{2}$, \\ Adel A. H. Abdel-Rahman ${ }^{3}$, Abdel-Aleem H. Abdel Aleem ${ }^{3}$, \\ Abdel Moneam Farag Eissa ${ }^{2}$ \\ ${ }^{1}$ Deanship of Scientific Research, Taif University, Alhawaya, Taif, KSA \\ ${ }^{2}$ Chemistry Department, Faculty of Science, Benha University, Benha, Egypt \\ ${ }^{3}$ Chemistry Department, Faculty of Science, Menaufia University, Shebin Elkom, Egypt \\ Email: mmharief@gmail.com, adelnassar63@science-mnf.eg, \\ aleem@science-mnf.eg,m.hasan@tu.edu.sa, \\ *magdah11uk@hotmail.com, amf_eissa@yahoo.com
}

How to cite this paper: Abdellattif, M.H., Arief, M.M.H., Abdel-Rahman, A.A.H., Aleem, A.-A.H.A. and Eissa, A.M.F. (2017) Green Synthesis of Novel 5-Arylazo-2-[(2S, 3S, 4R, 5R)-3, 4, 5-Trihydroxy-6-(Hydroxymethyl) Tetrahydro-2H-Pyran-2-Yloxy]-4, 6-Dimethyl 3-Nicotinonitrile. International Journal of Organic Chemistry, 7, 389-402. https://doi.org/10.4236/ijoc.2017.74031

Received: October 3, 2017

Accepted: December 12, 2017

Published: December 15, 2017

Copyright (๑) 2017 by authors and Scientific Research Publishing Inc. This work is licensed under the Creative Commons Attribution International License (CC BY 4.0).

http://creativecommons.org/licenses/by/4.0/

\begin{abstract}
Introduction: Pyridone derivatives played important roles in the last decade to approach many and different functionalities, especially as antitumor, antibacterial, anti-fungal, and many of pharmacological activities. Methodology: Novel compounds of 5-Arylazo-2-[(substituted)-3, 4, 5-trihydroxy-6-(hydroxymethyl) tetrahydro-2H-pyran-2-yloxy]-4, 6-dimethylnicotinonitrile, (3a-e), generally called (fluroarylazopyridine glucosides) were synthesised via green protocol, microwave. Results: The compounds were investigated by (IR, ${ }^{1} \mathrm{HNMR},{ }^{13} \mathrm{CNMR}$ and mass spectrometry). Where some of pharmacological activities like antibacterial and antifungal studies had been investigated and characterized. It was found that $3 \mathrm{a}-\mathrm{d}$ had characterized by high activities as antibacterial and antifungal. Where microwave synthetic methods were more efficient, gave higher products quantity, and more saving for time requirement and for using of much more solvents.
\end{abstract}

\section{Keywords}

Green Chemistry, Green Protocol, Pyridones, Fluroazo Compounds, Microwave 


\section{Graphical Abstract}

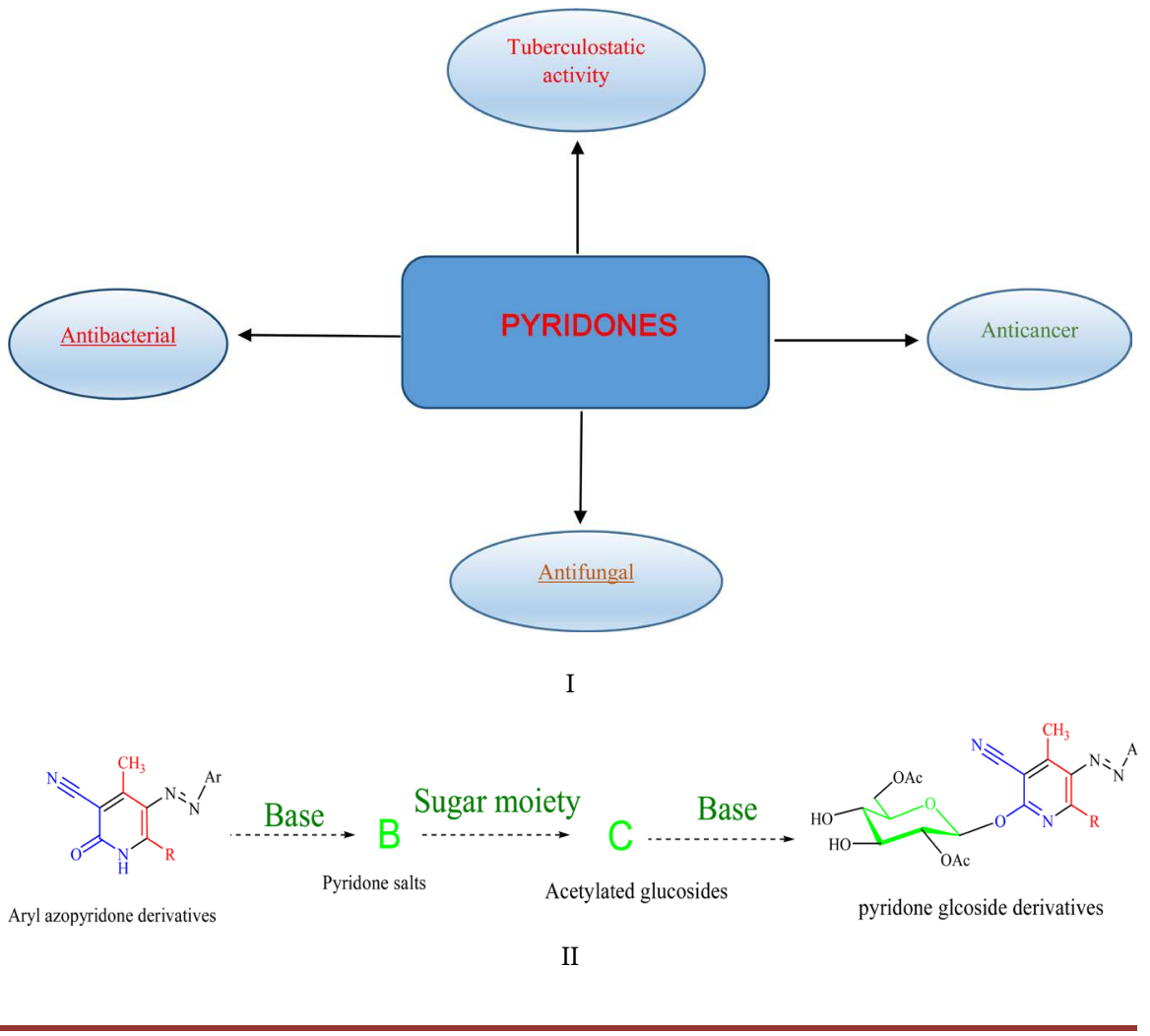

\section{Introduction}

Pyridine nucleus is one of the most interesting nucleus in organic synthesis. Many uses of pyridones derivatives were investigated in the recent decades especially fluorinated derivatives. One of the recent researches discovered that high tuberculostatic activity of pyridone was observed [1].

Also the amazing character of some pyridone is its high fluorescence activities which were used as molecular sensor of picric acid [2].

It has been of great importance in the exploring of some novel antimicrobial compounds in veterinary as well as human medicine worldwide.

Genetic mutation and propagation of drug resistance genes of microorganisms are a very great factor that being as a strong barrier in treating the infectious diseases for animal and human patients [3] [4].

The importance of the synthesis of novel derivatives of pyridone nucleosides is due to their strong affinity to treat many diseases, for example hepatitis, cancer and many of microbial infections [5] [6] [7] [8] [9].

Fluorinated derivatives of pyridones are of high significance in pharmaceutical and medicinal chemistry [10] [11]. Synthesis of poly substituted fluroarylazopyridone by using green protocol is of great effect in synthetic chemistry and also in pharmaceutical chemistry [12]. Actually in the molecule which contains fluorine atoms became of high change in its lipophilicity, which also affect and 
change the rate of transportation through lipid membranes [13].

Achievement of green and sustainable chemistry protocol instead of classical methods synthetic chemistry nowadays is of high interest, especially in synthesis of some novel substituted fluro pyridone derivatives (1a-e) and their nucleosides $(3 a-e)$.

\section{Results and Discussion}

\section{a) Chemistry}

General technique for green formation of arylazo pyridone glucosides had been used [14] [15].

Scheme 1. Represents the general method for synthesis of pyridine derivatives 1(a-e) where, Scheme 2. Represents the general method for synthesis of pyridine derivatives $2(\mathrm{a}-\mathrm{e})$ and $3(\mathrm{a}-\mathrm{e})$.

Where Simple, accurate, green procedure were be used in synthesis of 5-arylazo-2-[(2S，3S，4R，5R)-3，4，5-trihydroxy-6-(hydroxymethyl) tetrahydro-2H-pyran-2-yloxy]-4, 6-dimethylnicotinonitrile [16] [17] [18] [19].

5-arylazo-2-[(2S, 3S, 4R, 5R)-3, 4, 5-trihydroxy-6-(hydroxymethyl) tetrahydro-2H-pyran-2-yloxy]-4, 6-dimethylnicotinonitrile (3a-e) had been got in very good yields where microwave irradiation had been used.

In this method, a homogenous solid mixture of $2(1 \mathrm{H})$-pyridones (1a-e) and acetyled- $\alpha$-D-glucopyranose derivatives with silica gel was irradiated in MW for 2-3 minutes. For example, 3-cyano-4, 6-Dimethyl-5-arylazo-2( $1 H$-pyridinones (1a-e) [14] [15] were allowed to be reacted with acetylaed- $\alpha$-D-glucopyranosyl bromide derivative for 2 minutes to give (2a-e) in about $91 \%$ yield.<smiles>CC(=O)CC(C)=O</smiles><smiles>[Al-][N+]#[Ni]</smiles><smiles>CC(=O)C(=NN[Al])C(C)=O</smiles>
$\mathrm{Ar}$
MWI

3-5 min

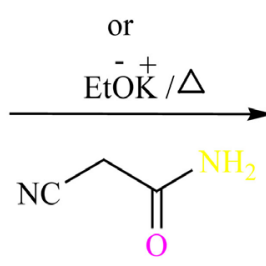<smiles>[3H][CH+]</smiles><smiles>Cc1[nH]c(=O)c(C#N)c(C)c1N=N[Al]</smiles>

Scheme 1. Synthesis of Pyridone derivatives 1a-e. 


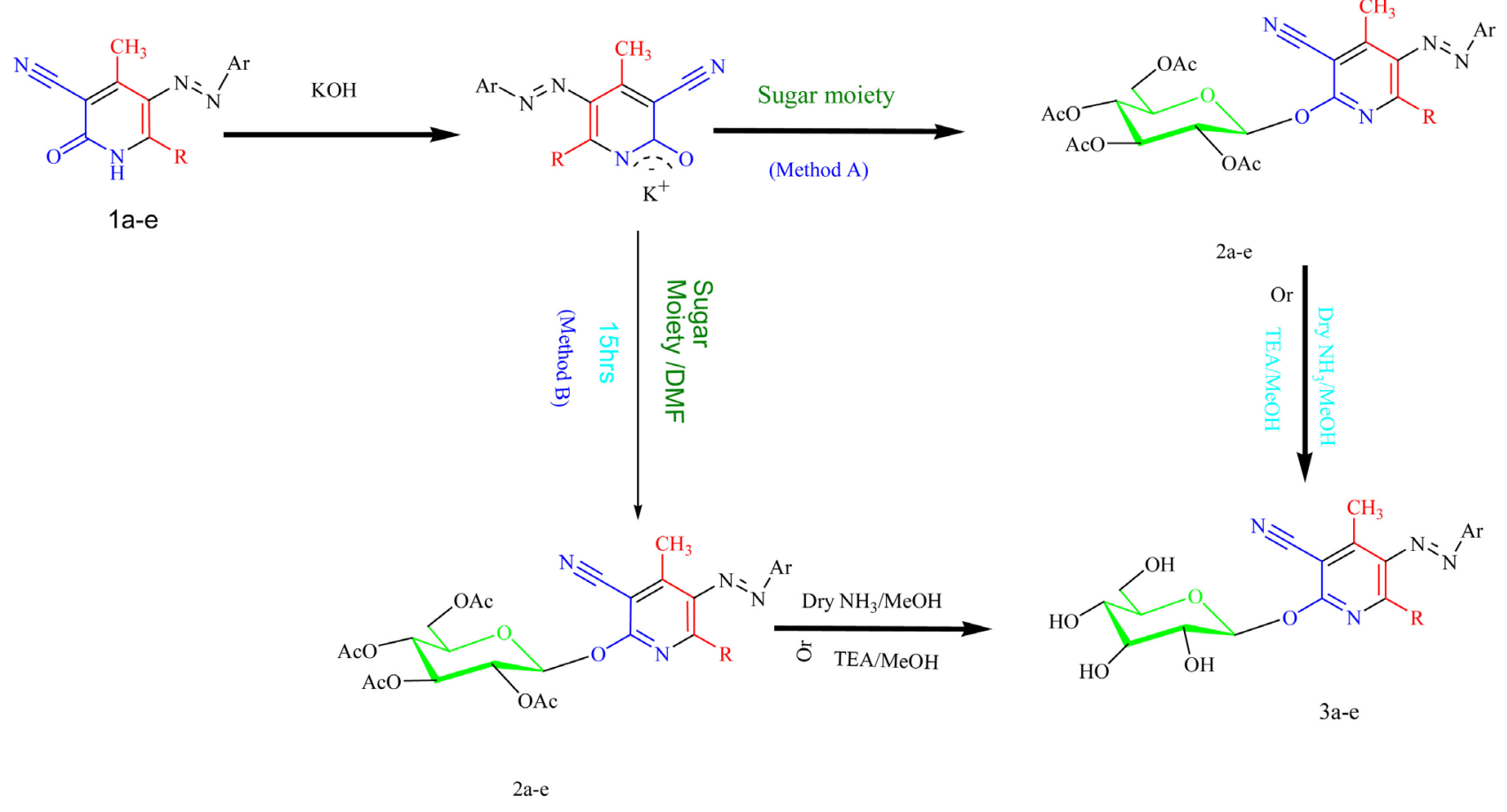

Scheme 2. Synthesis of Pyridone derivatives 2a-e \& 3a-e.

The same nucleosides, (2a-e) were be gained in very high yields by the reaction of the K-salt of pyridnone which was generated in situ, using potassium hydride and an activated sugar moiety. The K-salt of 3-cyano-4, 6-dimethyl-5-arylazo$2(1 \mathrm{H})$-pyridinones $(1 \mathrm{a}-\mathrm{e})$ were allowed to react with $\alpha$-bromoglucose in DMF for 15 hours to give (2a-e) in average $73 \%$ yield.

Deacetylation of 2a-e were almost done by treatment of alkali and although anhydrous media is useful to reduce the amount of alkali but catalytic reaction may be applied. But in fact a mixture of Triethylamine in $\mathrm{MeOH}$ and water be used in deacetylation or a mixture of methanol and dry ammonia were be also used.

Table 1 which illustrated the structure of the substituents of Compound 1 (a-e), where One could notice the difference between microwave method and conventional method in time of synthesis and the yield percentage of the product from Table 2. Also it had been noticed the difference between triethyl amine method and methanol and dry ammonia method in the yield percentage of the product as represented in Table 3.

The final structures of the expected compounds had been also represented in Table 4. Where the elemental analysis of the synthetic compounds had been illustrated in Table 5. Also ${ }^{1} \mathrm{HNMR},{ }^{13} \mathrm{CNMR}, \mathrm{Ms}-\mathrm{LC}$, and IR studies had been illustrated at Tables 6-9, respectively.

\section{b) Biology}

It has been of great importance in the exploring of some novel antimicrobial compounds in veterinary as well as human medicine worldwide. Genetic mutation and propagation of drug resistance genes of microorganisms are a very 
Table 1. Substituted Arylazopyridine glucosides 1a-e.

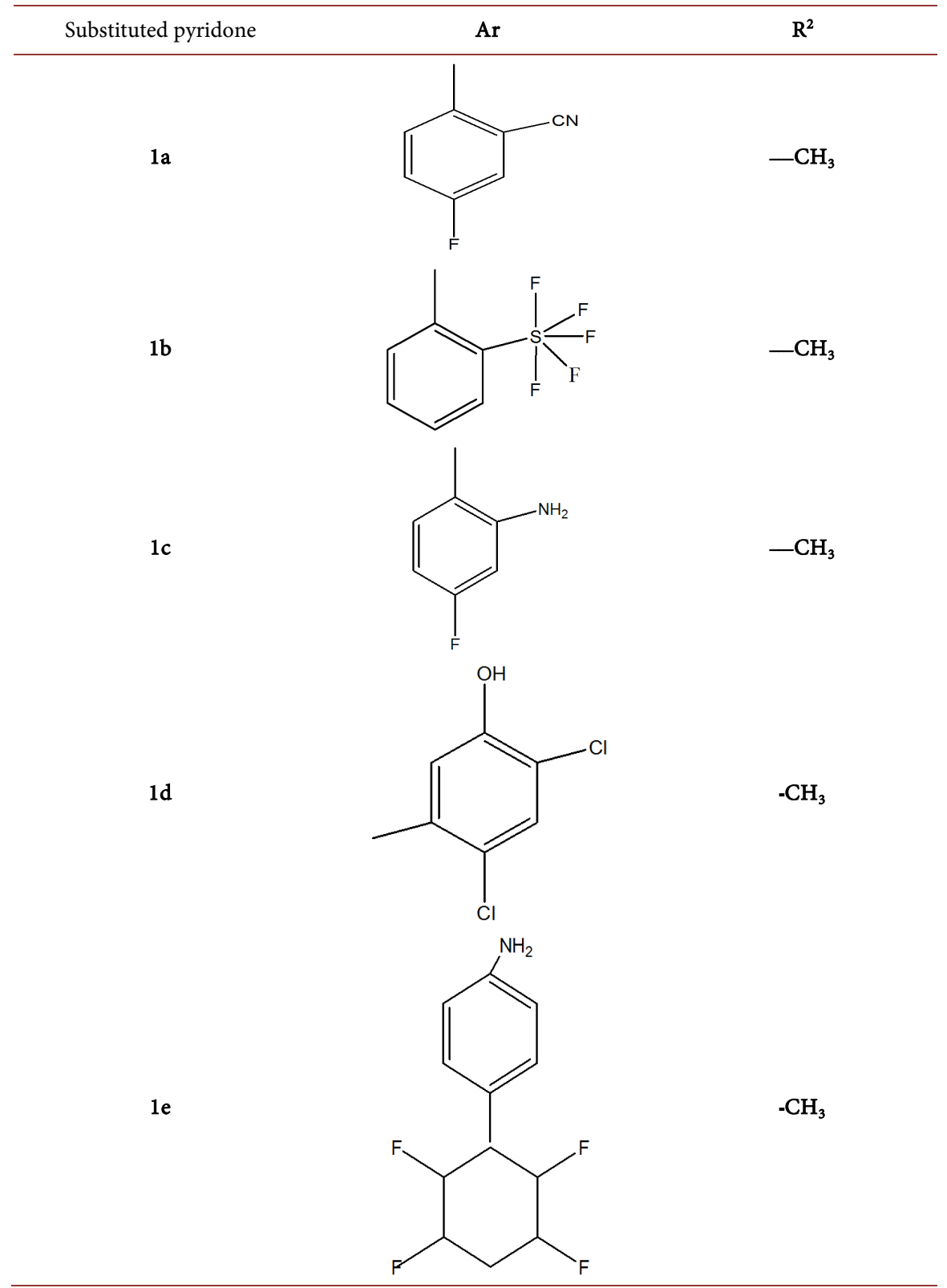

Table 2. Comparison between conventional methods and microwave methods for the Synthesis of Acetylated 5-arylazo-2-[(2S, 3S, 4R, 5R)-3, 4, 5-trihydroxy-6-(hydroxymethyl) tetrahydro-2H-pyran-2-yloxy]-4, 6-dimethyl 3-nicotinonitrile.

\begin{tabular}{ccccccc}
\hline \multirow{2}{*}{ Compound number } & $\mathbf{R}$ & $\mathrm{Ar}$ & \multicolumn{2}{c}{ Microwave method } & \multicolumn{2}{c}{ Conventional } \\
\cline { 5 - 8 } & & & time & yield & time & yield \\
\hline $2 \mathbf{a}$ & $-\mathrm{CH}_{3}$ & $\mathrm{C}_{7} \mathrm{H}_{3} \mathrm{~F}$ & 2 & 92 & 55 & 60 \\
$2 \mathbf{b}$ & $-\mathrm{CH}_{3}$ & $\mathrm{C}_{6} \mathrm{H}_{4} \mathrm{SF}_{5}$ & 2 & 94 & 48 & 69 \\
$2 \mathbf{2 c}$ & $-\mathrm{CH}_{3}$ & $\mathrm{C}_{6} \mathrm{H}_{5} \mathrm{NF}$ & 2 & 90 & 58 & 60 \\
$2 \mathrm{~d}$ & $-\mathrm{CH}_{3}$ & $\mathrm{C}_{6} \mathrm{H}_{2} \mathrm{Cl}_{2} \mathrm{NO}$ & 2 & 91 & 56 & 63 \\
$2 \mathbf{2 e}$ & $-\mathrm{CH}_{3}$ & $\mathrm{C}_{12} \mathrm{H}_{11} \mathrm{NF}_{4}$ & 2 & 93 & 55 & 60 \\
\hline
\end{tabular}


Table 3. Yeild \% percentage comparison of trimethylamine and dry ammonia methods for novel of nucleosides 3a-e.

\begin{tabular}{ccccc}
\hline Compound number & $\mathbf{R}=$ & $\mathbf{A r}=$ & Method A & Method B \\
\hline 3a & $-\mathrm{CH}_{3}$ & $\mathrm{C}_{7} \mathrm{H}_{3} \mathrm{~F}$ & 90 & 82 \\
3b & $-\mathrm{CH}_{3}$ & $\mathrm{C}_{6} \mathrm{H}_{4} \mathrm{SF}_{5}$ & 92 & 80 \\
$3 \mathbf{3 c}$ & $-\mathrm{CH}_{3}$ & $\mathrm{C}_{6} \mathrm{H}_{5} \mathrm{NF}$ & 91 & 81 \\
3d & $-\mathrm{CH}_{3}$ & $\mathrm{C}_{6} \mathrm{H}_{2} \mathrm{Cl}_{2} \mathrm{NO}$ & 92 & 83 \\
3e & $-\mathrm{CH}_{3}$ & $\mathrm{C}_{12} \mathrm{H}_{11} \mathrm{NF}_{4}$ & 92 & 80 \\
\hline
\end{tabular}

Table 4. Structure formulae for compounds 1a-e \& 3a-e.

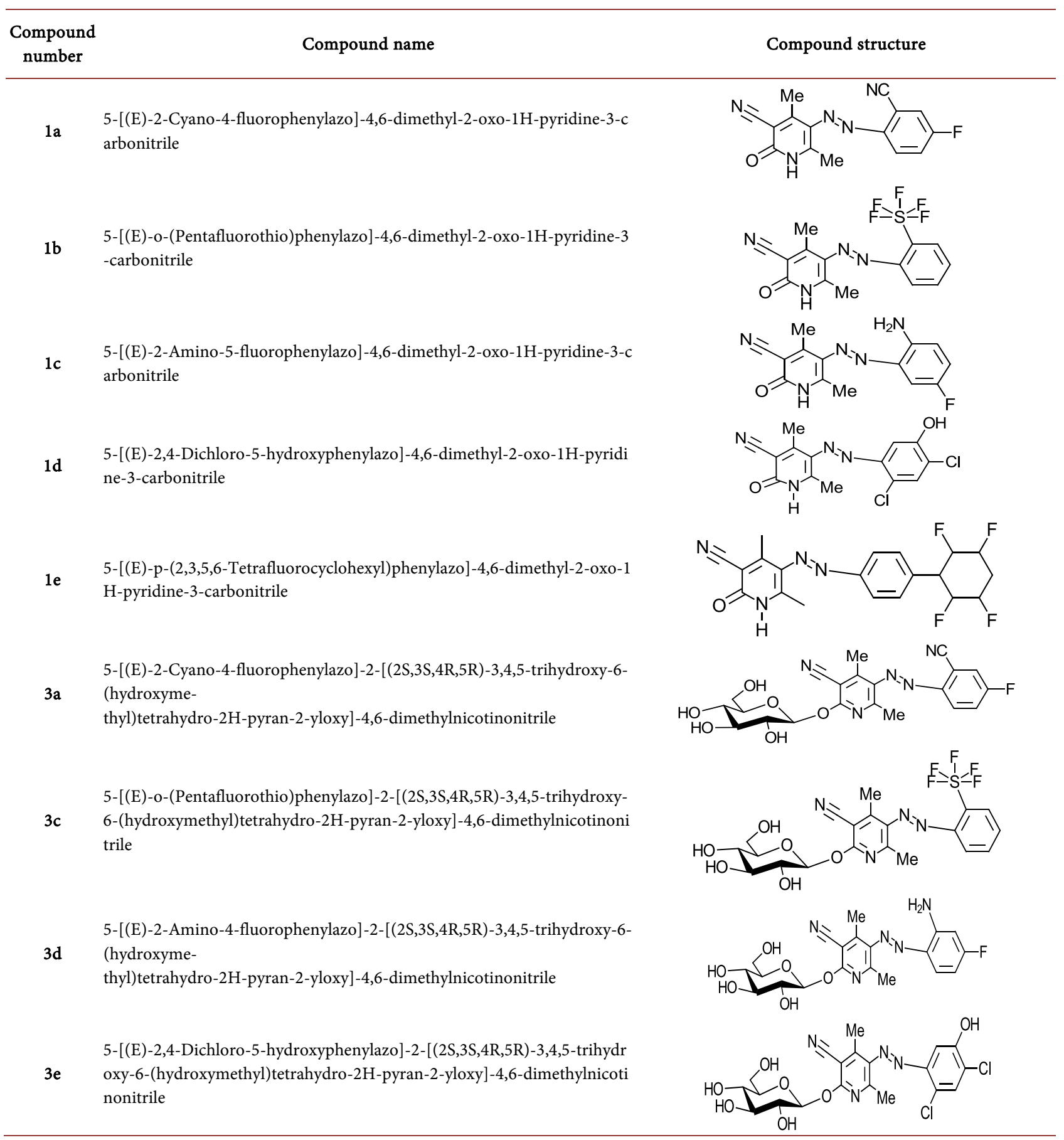


Table 5. The Elemental analysis of synthesized compounds.

\begin{tabular}{|c|c|c|c|c|c|c|c|c|c|}
\hline \multirow{2}{*}{ Compd. } & \multirow{2}{*}{$\mathrm{Mp} \cdot\left({ }^{\circ} \mathrm{C}\right)$} & \multirow{2}{*}{ Formula (Mwt) } & \multirow{2}{*}{ Solvent of crystallization } & \multicolumn{6}{|c|}{ Analysis \% Calcd. (Found) } \\
\hline & & & & $\mathrm{C}$ & $\mathrm{H}$ & $\mathrm{N}$ & $\mathrm{Cl}$ & $\mathrm{F}$ & S \\
\hline $1 \mathrm{a}$ & 456 & $\mathrm{C}_{15} \mathrm{H}_{10} \mathrm{FN}_{5} \mathrm{O}$ & Ethanol/DME & 61.01 & 3.41 & 23.72 & & 6.43 & \\
\hline $1 b$ & 240.76 & $\mathrm{C}_{14} \mathrm{H}_{11} \mathrm{~F}_{5} \mathrm{~N}_{4} \mathrm{OS}$ & Ethanol/DME & 44.45 & 2.93 & 14.81 & & 25.11 & 8.48 \\
\hline $1 c$ & 399.66 & $\mathrm{C}_{14} \mathrm{H}_{12} \mathrm{FN}_{5} \mathrm{O}$ & Ethanol/DME & 58.94 & 4.24 & 24.55 & & 6.66 & \\
\hline $1 d$ & 487.37 & $\mathrm{C}_{14} \mathrm{H}_{10} \mathrm{Cl}_{2} \mathrm{~N}_{4} \mathrm{O}_{2}$ & Ethanol/DME & 49.87 & 2.99 & 16.62 & 21.03 & & \\
\hline $1 e$ & 363.69 & $\mathrm{C}_{20} \mathrm{H}_{18} \mathrm{~F}_{4} \mathrm{~N}_{4} \mathrm{O}$ & Ethanol/DME & 59.11 & 4.46 & 13.79 & & 18.7 & \\
\hline $3 a$ & 794 & $\mathrm{C}_{21} \mathrm{H}_{20} \mathrm{FN}_{5} \mathrm{O}_{6}$ & Ethanol/DME & 55.14 & 4.41 & 15.31 & & 4.15 & \\
\hline $3 b$ & 578 & $\mathrm{C}_{20} \mathrm{H}_{21} \mathrm{~F}_{5} \mathrm{~N}_{4} \mathrm{O}_{6} \mathrm{~S}$ & Ethanol/DME & 44.45 & 3.92 & 10.73 & & 17.58 & 5.93 \\
\hline $3 c$ & 737 & $\mathrm{C}_{20} \mathrm{H}_{22} \mathrm{FN}_{5} \mathrm{O}_{6}$ & Ethanol/DME & 53.69 & 4.96 & 15.72 & & 4.25 & \\
\hline $3 d$ & 825 & $\mathrm{C}_{20} \mathrm{H}_{20} \mathrm{Cl}_{2} \mathrm{~N}_{4} \mathrm{O}_{7}$ & Ethanol/DME & 48 & 3.64 & 16.86 & & & 9.65 \\
\hline $3 e$ & 701 & $\mathrm{C}_{26} \mathrm{H}_{28} \mathrm{~F}_{4} \mathrm{~N}_{4} \mathrm{O}_{6}$ & Ethanol/DME & 54.93 & $4 . .04$ & 11.22 & 14.3 & & \\
\hline
\end{tabular}

Table 6. ${ }^{1} \mathrm{H}$ NMR spectrum data of the novel compounds.

\begin{tabular}{|c|c|}
\hline $\begin{array}{l}\text { Compound } \\
\text { number }\end{array}$ & Spectral1H NMR data \\
\hline $1 \mathrm{a}$ & $\delta 1.77\left(\mathrm{~s}, \mathrm{CH}_{3}, 3 \mathrm{H}\right), 1.7\left(\mathrm{~s}, \mathrm{CH}_{3}, 3 \mathrm{H}\right), 7.5(\mathrm{q}, 3 \mathrm{H}, \mathrm{Ar}-\mathrm{H})$ \\
\hline $1 \mathrm{~b}$ & $\delta 1.77\left(\mathrm{~s}, \mathrm{CH}_{3}, 3 \mathrm{H}\right), 1.7\left(\mathrm{~s}, \mathrm{CH}_{3}, 3 \mathrm{H},\right), 7.2-7.5(\mathrm{~m}, 4 \mathrm{H}, \mathrm{Ar}-\mathrm{H})$ \\
\hline $1 c$ & $\delta 1.9\left(\mathrm{~s}, \mathrm{CH}_{3}, 3 \mathrm{H}\right), 1.77\left(\mathrm{~s}, \mathrm{CH}_{3}, 3 \mathrm{H}\right), 6.9(\mathrm{q}, 3 \mathrm{H}, \mathrm{Ar}-\mathrm{H})$ \\
\hline $1 \mathrm{~d}$ & $\delta 1.9\left(\mathrm{~s}, \mathrm{CH}_{3}, 3 \mathrm{H}\right), 1.77\left(\mathrm{~s}, \mathrm{CH}_{3}, 3 \mathrm{H}\right), 7.4(\mathrm{~d}, 2 \mathrm{H}, \mathrm{Ar}-\mathrm{H})$ \\
\hline $1 \mathrm{e}$ & $\Delta 1.77\left(\mathrm{~s}, \mathrm{CH}_{3}, 3 \mathrm{H}\right), 1.7\left(\mathrm{~s}, \mathrm{CH}_{3}, 3 \mathrm{H}\right), 2.3\left(\mathrm{~m}, \mathrm{CH}_{2}, 2 \mathrm{H}\right), 5.4(\mathrm{~m}, \mathrm{CH}, 4 \mathrm{H}), 3.98(\mathrm{~m}, \mathrm{H}, \mathrm{CH}), 8.9(\mathrm{~s}, \mathrm{H}, \mathrm{Ar}-\mathrm{H})$ \\
\hline $3 \mathrm{a}$ & $\begin{array}{l}\delta\left(\mathrm{s}, \mathrm{CH}_{3}, 3 \mathrm{H}\right), 1.7\left(\mathrm{~s}, \mathrm{CH}_{3}, 3 \mathrm{H}\right), 7.5(\mathrm{~m}, 3 \mathrm{H}, \mathrm{Ar}-\mathrm{H}), 3.4\left(\mathrm{~d}, 2 \mathrm{H},-\mathrm{CH}_{2}{ }^{\prime \prime \prime \prime \prime)}\right), 6.9(\mathrm{~d}, 1 \mathrm{H},-\mathrm{CH}), 2.4(\mathrm{~m}, \mathrm{H}, \mathrm{OH}), 4.4\left(\mathrm{~m}, 3 \mathrm{H}, \mathrm{CH}^{\prime \prime},\right. \\
\left.\mathrm{CH}^{\prime \prime \prime}, \mathrm{CH}^{\prime \prime \prime \prime)}\right)\end{array}$ \\
\hline $3 b$ & 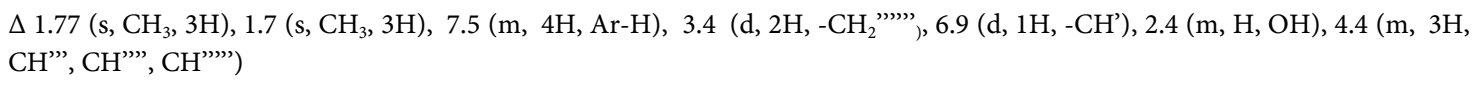 \\
\hline $3 c$ & 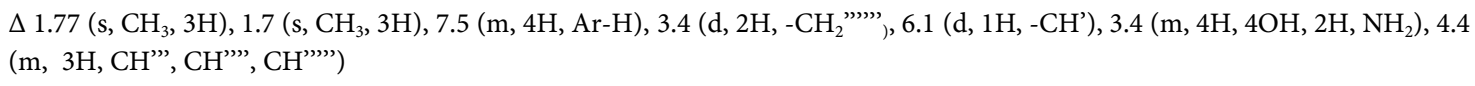 \\
\hline $3 \mathrm{~d}$ & $\begin{array}{l}\delta 1.77\left(\mathrm{~s}, \mathrm{CH}_{3}, 3 \mathrm{H}\right), 1.7\left(\mathrm{~s}, \mathrm{CH}_{3}, 3 \mathrm{H}\right), 6.7-7.5(\mathrm{~d}, \mathrm{~d}, 2 \mathrm{H}, \mathrm{Ar}-\mathrm{OH}), 2.9(\mathrm{~m}, \mathrm{H}, 5 \mathrm{OH}), 6.1\left(\mathrm{~d}, 1 \mathrm{H},-\mathrm{CH}^{\prime}\right), 3.37-4.4\left(\mathrm{~m}, 3 \mathrm{H}, \mathrm{CH}^{\prime \prime},\right. \\
\left.\mathrm{CH}^{\prime \prime \prime \prime}, \mathrm{CH}^{\prime \prime \prime \prime}\right)\end{array}$ \\
\hline $3 e$ & $\begin{array}{l}\delta 1.77\left(\mathrm{~s}, \mathrm{CH}_{3}, 3 \mathrm{H}\right), 1.7\left(\mathrm{~s}, \mathrm{CH}_{3}, 3 \mathrm{H}\right), 7.5(\mathrm{q}, 3 \mathrm{H}, \mathrm{Ar}-\mathrm{H}), 7.2\left(\mathrm{~s}, 2 \mathrm{H},-\mathrm{CH}_{2}^{\prime \prime \prime \prime \prime)}\right), 6.9\left(\mathrm{~d}, 1 \mathrm{H},-\mathrm{CH}^{\prime \prime}\right), 2.4(\mathrm{~m}, \mathrm{H}, \mathrm{OH}), 4.4(\mathrm{q}, 4 \mathrm{H}, \mathrm{CH} \text {, } \\
\left.\mathrm{CH}^{\prime \prime \prime}, \mathrm{CH}^{\prime \prime \prime \prime}, \mathrm{CH}^{\prime \prime \prime \prime \prime)}\right)\end{array}$ \\
\hline
\end{tabular}

Table 7. ${ }^{13} \mathrm{C}$ NMR spectrum data of the novel compounds.

\begin{tabular}{|c|c|}
\hline $\begin{array}{c}\text { Compound } \\
\text { number }\end{array}$ & Spectral 13C NMR data \\
\hline $1 \mathrm{a}$ & 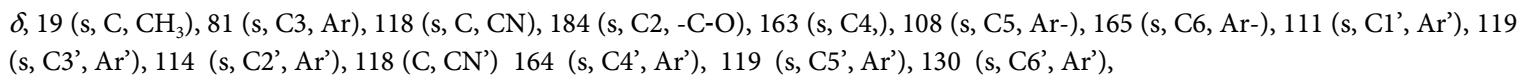 \\
\hline $1 b$ & 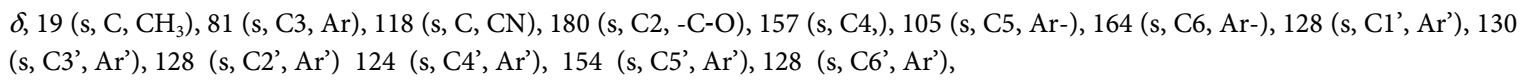 \\
\hline $1 c$ & 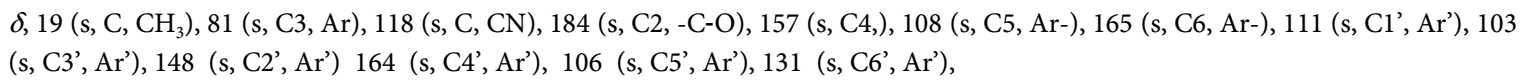 \\
\hline
\end{tabular}




\section{Continued}

$1 \mathrm{~d}$

$\delta, 19$ (s, C, $\mathrm{CH}_{3}$ ), 81 (s, C3, Ar), 118 (s, C, CN), 184 (s, C2, -C-O), 157 (s, C4,), 105 (s, C5, Ar-), 164 (s, C6, Ar-), 128 (s, C1', Ar'), 130 (s, C3', Ar'), 128 (s, C2', Ar') 124 (s, C4', Ar'), 154 (s, C5', Ar'), 118 (s, C6', Ar').

$1 e$ $\delta, 19$ (s, C, $\mathrm{CH}_{3}$ ), 118 (s, C, CN), 153 (s, C, -C=O), 117 (s, C3, Ar-), 157 (s, C4, Ar-), 115 (s, C5, Ar-), 163 (s, C6, Ar-), 128 (s, C1', Ar'), 130 (s, C3', C5', Ar'), 128 (s, C2', C6', Ar') 124 (dd, C4', Ar'), 31 (m, C1'”), 92 (m, C2"', C6”') , 85 (m, C3"', C5'”) 27 (C4"')

$\delta, 19$ (s, C, $\mathrm{CH}_{3}$ ), 81 (s, C3, Ar), 118 (s, C, CN), 184 (s, C2, -C-O), 163 (s, C4,), 108 (s, C5, Ar-), 165 (s, C6, Ar-), 111 (s, C1', Ar'), 119

3a (s, C3', Ar'), 114 (s, C2', Ar'), 118 (C, CN') 164 (s, C4', Ar'), 119 (s, C5', Ar'), 130 (s, C6', Ar'), 131 (s, C1"), 67 (s, C2”), 67 (s, C3”), 67 (s, C4"), 81 (s, C5"), 60 (s, C6"), $128\left(\mathrm{~s}, \mathrm{Cl}^{\prime}, \mathrm{Ar}^{\prime}\right), 130$ (s, C3', Ar'), 128 (s, C2', Ar') 124 (s, C4', Ar'), 154 (s, C5', Ar'), 128 (s, C6', Ar'), 131 (s, C1'), 67 (s, C2'), 67 (s, C3”), 67 (s, C4'), 81 (s, C5"), 60 (s, C6"),

$3 c$ $\delta, 19$ (s, C, $\mathrm{CH}_{3}$ ), 81 (s, C3, Ar), 118 (s, C, CN), 184 (s, C2, -C-O), 157 (s, C4,), 108 (s, C5, Ar-), 165 (s, C6, Ar-), 111 (s, Cl', Ar'), 103 (s, C3', Ar'), 148 (s, C2', Ar') 164 (s, C4', Ar'), 106 (s, C5', Ar'), 131 (s, C6', Ar'), 131 (s, C1"), 67 (s, C2”), 67 (s, C3"), 67 (s, C4"), 81 (s, C5"), 60 (s, C6"),

$\delta, 19$ (s, C, $\mathrm{CH}_{3}$ ), 81 (s, C3, Ar), 118 (s, C, CN), 184 (s, C2, -C-O), 157 (s, C4,), 105 (s, C5, Ar-), 164 (s, C6, Ar-), 128 (s, C1', Ar'), 130 (s, C3', Ar'), 128 (s, C2', Ar') 124 (s, C4', Ar'), 154 (s, C5', Ar'), 118 (s, C6', Ar'), 131 (s, C1"), 67 (s, C2”), 67 (s, C3”), 67 (s, C4”), 81 (s, C5"), 60 (s, C6"),

$\delta, 19$ (s, C, $\mathrm{CH}_{3}$ ), 81 (s, C3, Ar), 118 (s, C, CN), 184 (s, C2, -C-O), 157 (s, C4,), 105 (s, C5, Ar-), 164 (s, C6, Ar-), 128 (s, C1', Ar'), 130 (s, C3', C5', Ar'), 128 (s, C2', C6', Ar') 124 (dd, C4', Ar'), 131 (s, C1"), 67 (s, C2"), 67 (s, C3"), 67 (s, C4”), 81 (s, C5"), 60 (s, C6”), 31

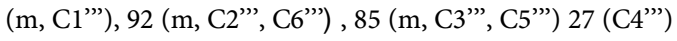

Table 8. LC/Ms Fragmentation spectrum data of synthesized compounds of Scheme 13.

\begin{tabular}{ccc}
\hline Compound & $\mathbf{m} / \mathbf{z}$ & Abundance\% \\
\hline la & 295.27 & 98 \\
1b & 378.32 & 99 \\
1c & 285.28 & 97 \\
1d & 337.16 & 99 \\
le & 406 & 99 \\
3a & 457.41 & 96 \\
3b & 540.5 & 98 \\
3c & 472 & 97 \\
3d & 498 & 98 \\
3e & 568 & 99 \\
\hline
\end{tabular}

Table 9. IR spectrum data for the synthesized compounds Scheme 12.

\begin{tabular}{cc}
\hline Compound & IR $v \mathrm{~cm}^{-1}$ \\
\hline $1 \mathrm{a}$ & $3123(\mathrm{NH}), 2220(\mathrm{CN}), 1646(\mathrm{CO})$ \\
lb & $3226(\mathrm{NH}), 2215(\mathrm{CN}), 1717(\mathrm{CO})$. \\
$1 \mathrm{c}$ & $3300,3400\left(\mathrm{NH}_{2}\right) ; 1645(\mathrm{C}=\mathrm{O}) 3195(\mathrm{NH}), 2223(\mathrm{CN})$ \\
ld & $3300(\mathrm{OH}), 2230(\mathrm{CN}), 1690(\mathrm{CO}), 3500(\mathrm{NH})$ \\
$1 \mathrm{e}$ & $3200(\mathrm{OH}), 1640(\mathrm{CO}), 3300(\mathrm{NH}), 2250(\mathrm{CN})$ \\
$3 \mathrm{a}$ & $3300,3400\left(\mathrm{NH}_{2}\right) ; 1645(\mathrm{C}=\mathrm{O}) 3195(\mathrm{NH}), 2223(\mathrm{CN})$ \\
$3 \mathrm{~b}$ & $3100(\mathrm{NH}), 1650(\mathrm{CO}), 2228(\mathrm{CN})$ \\
$3 \mathrm{c}$ & $3195(\mathrm{NH}), 2223(\mathrm{CN}), 1645(\mathrm{C}=\mathrm{O})$ \\
$3 \mathrm{~d}$ & $3300(\mathrm{OH}) ; 1680(\mathrm{C}=\mathrm{O}) ; 2224(\mathrm{CN}) ; 3455(\mathrm{NH})$ \\
3e & $3300(\mathrm{NH}), 2225(\mathrm{CN}), 1650(\mathrm{C}=\mathrm{O})$ \\
\hline
\end{tabular}


great factor that being as a strong barrier in treating the infectious diseases for animal and human patients.

All investigated compounds show different antibacterial and antifungal activities, these results were be due to the newly derivatives formed from fluroazo pyridone and their glucosides.

The most active compounds were 1a, $3 \mathrm{a}, 1 \mathrm{c}, 3 \mathrm{c}$ although most of them showed good activity.

One could notice that Table 10, represented the antibacterial and the anti-fungal effects of the new synthetic compounds. Where Table 11 represented the strain of organism.

\section{Methodolgy}

\subsection{Chemistry}

3.1.1. General Coupling Procedures of Synthesis of Acetylated-Arylazo2-[(2S, 3S, 4R, 5R)-3, 4, 5-Trihydroxy-6-(Hydroxymethyl) Tetrahydro-2H-Pyran-2-Yloxy]-4,6 Dimethylnicotinonitrile

Microwave synthesis will be performed using CEM Microwave system. Melting

Table 10. Virtual screening for in vitro Antibacterial and antifungal of the novel fluroarylazopyridin-2-one derivatives.

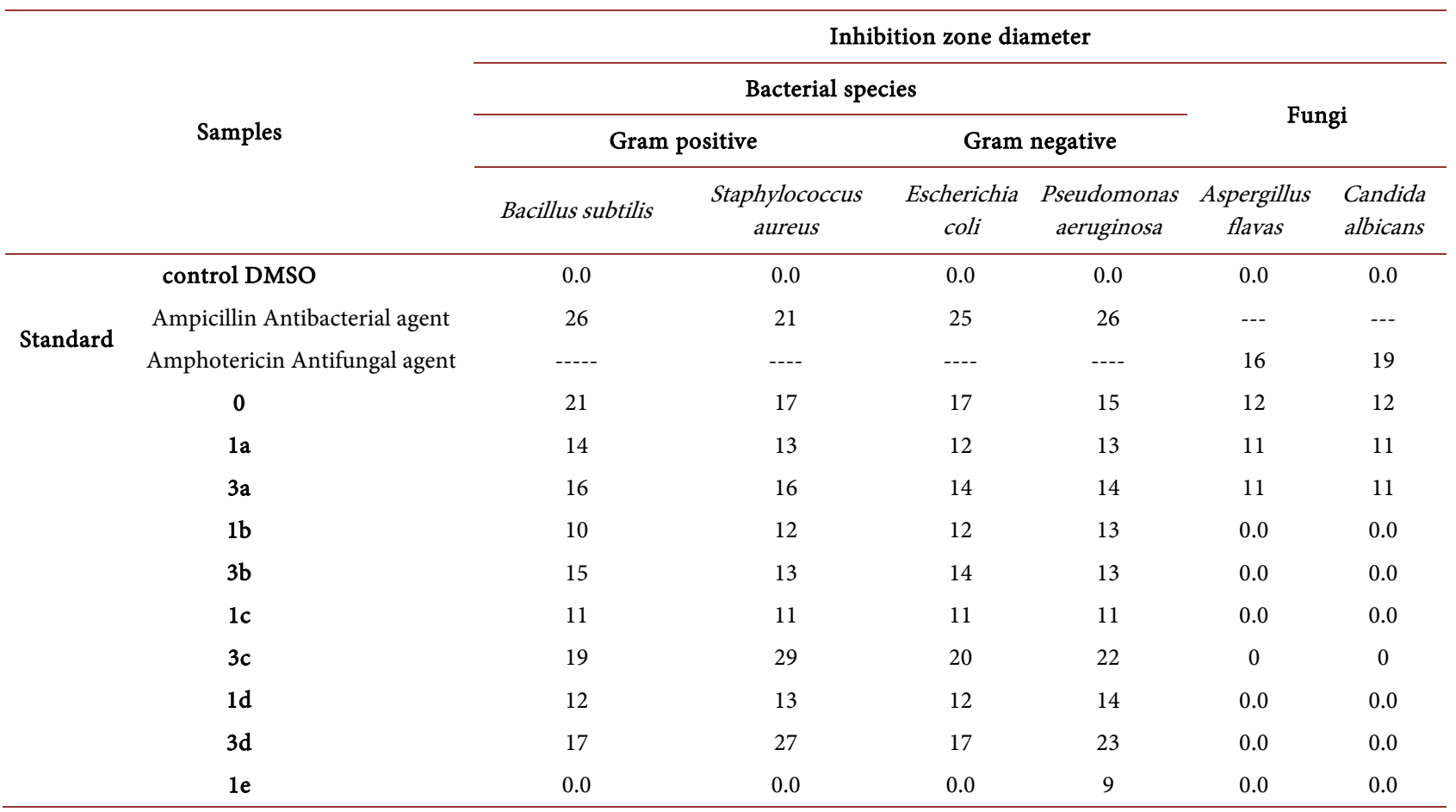

Table 11. The type strain of microorganisms.

\begin{tabular}{ccc}
\hline Microorganism & Gram Reaction & ATCC \\
\hline Escherichia coli & $\mathrm{G}^{-}$ & 11,775 \\
Staphylococcus aureus & $\mathrm{G}^{+}$ & 12,600 \\
Candida albicans & Fungus & 7102 \\
Aspergillus flavus & & Link \\
\hline
\end{tabular}


points will be determined on (Pyrex capillary) Gallenkamp apparatus. Infrared spectra will be recorded with a Thermo Nicolet Nexus 470 FT-IR spectrometer in the range $4000-400 \mathrm{~cm}^{-1}$ using potassium bromide disks. ${ }^{1} \mathrm{H}-\mathrm{NMR}$ spectra, ${ }^{13} \mathrm{C}-\mathrm{NMR}$ spectra will be obtained on Varian Gemini 400 and $200 \mathrm{MHz}$ FT NMR spectrometer in $\mathrm{CDCl}_{3}$ and DMSO- $d_{6}$; chemical shifts will be recorded in $\delta$ (ppm) units, relative to $\mathrm{Me}_{4} \mathrm{Si}$ as an internal standard. The mass spectra will be recorded on Shimadzu LCMS-QP 800 LC-MS and AB-4000 Q-trap LC-MS/MS. Thin-layer chromatography (TLC) will be carried out on pre-coated Merck silica gel $\mathrm{F}_{254}$ plates. Column chromatography will be performed on a Merck silica gel. The reagents will be purchased from Aldrich and used without further purification.

\section{a) Green Microwave method}

A solution of 2(1H)-pyridones (1a-e) $(10 \mathrm{mmol})$ and acetylated- $\alpha$-D-glucopyranose derivatives (11 mmol, $4.29 \mathrm{~g}$ ) had been prepared by dissolving in methylene chloride/methanol (80/20) then silica gel (200 - 400 mesh) mixture. Then the excess solvent had been subjected to vaporization to be removed. The dried residue had been transferred into a vial and subjected to microwave irradiation for 2 - 3 minutes using CEM Microwave system. The product will be purified using column to gain (2a-e).

\section{b) Conventional method}

A solution of $2(1 H)$-pyridone $(1 \mathrm{a}-\mathrm{e})(10 \mathrm{mmol})$ in DMF $(15 \mathrm{ml})$ had been mixed with potassium hydride $(4.76 \mathrm{mmol})$ under nitrogen they had been stirred at $60^{\circ} \mathrm{C}$. After $2 \mathrm{~h}$, the acetylated glucopyranosyl bromide (5) (15 mmol,), had been added and the solution had been stirred at normal temperature for $15 \mathrm{~h}$. The solvent had been evaporated and reminder prepared material had been partitioned between $\mathrm{CHCl}_{3}(30 \mathrm{~mL})$ and water $(30 \mathrm{~mL})$. Mixed organic extracts had been dried on $\left(\mathrm{Na}_{2} \mathrm{SO}_{4}\right)$, filtered and vaporized until complete dryness. The crude synthetic compounds had been dried and purified using column chromatography to gain the compounds (2a-e).

\subsubsection{General Procedure for Nucleoside Deacetylation}

\section{a) Triethyl amine method}

Triethylamine $(1.0 \mathrm{ml})$ had been mixed with glucosides solution of (2a-e). (1 $\mathrm{m}$ mol) in (10 $\mathrm{ml}$ methanol and few drops of water). The mixture had been stirred for 15 hours at normal. Reduced pressure had been applied for the vaporization and the residue was evaporated with methanol until triethylamine had removed. The synthetic compounds had been crystallized using suitable solvents to get compounds (3a-e).

\section{b) Methanol and dry ammonia}

Dry methanol $(20 \mathrm{ml})$ solution of protected glucosides $(0.5 \mathrm{~g})$ of at $0^{\circ} \mathrm{C}(2 \mathrm{a}-\mathrm{e})$ had been treated by dry ammonia for 25 minutes. The reaction mixture had been stirred till it had done and finally had been investigated by TLC. Reduced pressure had been applied to get the resultant products concentrated till crude solid had been reached. A solution (chloroform: methanol, 20:1), and silica gel chro- 
matography had been applied to the products for purification. Methanol had been applied for crystallization to gain (3a-e).

\subsection{Biology}

A method of Kirby-Bauer had been used for the determination of antimicrobial activity for novel fluroazopyridone compounds [20].

This had been carried out using special concentration of the test bacteria/fungi and had been grown up in a certain concentration of fresh media and then left it to grow up to $10^{8}$ cells $/ \mathrm{mL}$ for bacteria $10^{5}$ cells $/ \mathrm{mL}$ for fungi [21].

Onto agar plates a concentration of $100 \mu \mathrm{l}$ of bacterial of fungal suspension had been spread according to the broth for their maintenance. Each isolated colony for any organism used had played a pathological effect on primary and tested. Utility of disc diffusion method that many media were available, NCCLS [22] gave a recommendation of using Mueller-Hinton agar because of [23] [24] their reproducibility in good batch-to-batch.

An approved standard method (M38-A) disc diffusion method which had been applied to filamentous fungi tested [24] [25] [26] to evaluate the pathological activity of filamentous fungi to antifungal agents. Using an approved standard method (M44-P) had been used for disc diffusion method for yeasts developed by [25]. Aspergillus flavus used as an example of fungi had been incubated at $25^{\circ} \mathrm{C}$ for 48 hours. Staphylococcus aureus, Bacillus subtilis used as an example of Gram positive bacteria, where Escherichia coli, Pseudomonas aeuroginosa used as an example of Gram negative bacteria, both had been incubated at $35^{\circ} \mathrm{C}-37^{\circ} \mathrm{C}$ for $24-48$ hours.

Where; Candida albicans used as yeast had been incubated at $30^{\circ} \mathrm{C}$ for $24-48$ hours.

The inhibition zones diameter had been measured in millimetres [20].

A positive control for microbial activity had been served by standard discs of Ampicillin (Antibacterial agent), Amphotericin B (Antifungal agent). A negative control had been achieved by immersing of filter discs in $10 \mu \mathrm{l}$ of solvent (distilled water, chloroform, DMSO). Meuller-Hinton agar was used for tested the composition and $\mathrm{pH}$.

In addition a factor had been considered in the disc diffusion method was the depth of the agar in the plate. This method was a very known one and well documented and standard zones of inhibition had been determined for susceptible and resistant values. Blank paper disks (Schleicher \& Schuell, Spain) with a diameter of $8.0 \mathrm{~mm}$ were impregnated $10 \mu \mathrm{l}$ of tested concentration of the stock solutions. An impregnated filter paper disc with a tested chemical had been put on agar and the novel chemical compound had been diffused from the disc into the agar. By this diffusion the chemical compound had been transferred in the agar only around the disc. The size of the area of chemical infiltration around the disc had been determined by the chemical compound solubility and its molecular size. Bacteria organism had not been grown up when placed in the agar 
which contains the chemical compounds under investigation. This area of no growth around the disc had been named as a "Zone of inhibition" or "Clear zone". The diffusion, the zone diameters were measured with the National Committee for Clinical Laboratory Standards. Good alternatives methods had been characterized such as E-test and disk diffusion and they were simpler and faster techniques [26].

\section{References}

[1] Goryaeva, M.V., Burgart, Y.V., Kudyakova, Y.S., Ezhikova, M.A., Kodess, M.I. and Saloutin, V.I. (2017) Synthesis of Pyridone Derivatives from 7-Hydroxy-7-polyfluoroalkylhexahydroimidazo[1,2-a]pyridin-5-ones. European Journal of Organic Chemistry, 2017, 3986-3991. https://doi.org/10.1002/ejoc.201700683

[2] Luo, T.T., Li, Y.Q., Xu, Y.X., Zhang, S.T., Wang, Y.J., Kou, X.M. and Xiao, D. (2017) Rapid Synthesis of a Hyperfluorescence 2-Pyridone Derivative as a Fluorescent Molecular Sensor for Picric Acid. Sensors and Actuators B, 253, 231-238. https://doi.org/10.1016/j.snb.2017.06.080

[3] Rateb, N.M., El-Deab, H.A. and Abdou, I.M. (2013) Antimicrobial Evaluation of New Synthesize Pyridine Nucleosides under Solvent-Free Conditions. Nucleosides, Nucleotides and Nucleic Acids, 32, 493-509. https://doi.org/10.1080/15257770.2013.827206

[4] Romeo, R., Carnovale, C., Salvatore, V., Romeo, G., Macchi, B., Frezza Caterina, F. and Pistar'a, V. (2012) Truncated Phosphonated C-1'-branched N,O-Nucleosides: A New Class of Antiviral Agents. Bioorganic \& Medicinal Chemistry, 20, 3652-3657. https://doi.org/10.1016/j.bmc.2012.03.047

[5] Abdel Motaal, E.A., El-Gaby, M.S.A. and Salem, M.A. (2015) Design, Synthesis and Anticancer Activity of New 3-cyano-2 (1H) -pyridone and cyanopyridine-2-(1H)-thione Derivatives. Oriental Journal of Chemistry, 31, 875-884. https://doi.org/10.13005/ojc/310230

[6] Abdellattif, M.H., Maghrabi, I.A., Areef, M.M.H., El-Deab, H.A., Mouneir, S.M. and Belal, A. (2016) Efficient Microwave-Assisted Solvent-Free Synthesis and Molecular Docking Studies of 2-pyridone derivatives as Anticancer Agents and Evaluation of Cytotoxic Effects. Journal of Advances in Chemistry, 12, 4351-4364.

[7] Abadi, A.H., Ibrahim, T.M., Abouzid, K.M., Lehmann, J., Tinsley, H.N., Gary, B.D. andPiazza, G.A. (2009) Design, Synthesis and Biological Evaluation of Novel Pyridine Derivatives as Anticancer Agents and Phosphodiesterase 3 Inhibitors. Bioorganic \& Medicinal Chemistry, 17, 5974-5982.

https://doi.org/10.1016/j.bmc.2009.06.063

[8] Khidre, R.E., El-Gogary, S.R. and Mostafa, M.S. (2017) Design, Synthesis, and Antimicrobial Evaluation of some Novel Pyridine, Coumarin, and Thiazole Derivatives. Journal of Heterocyclic Chemistry, 54, 2511. https://doi.org/10.1002/jhet.2854

[9] Mahmoud, M.R., El-Azm, F.S.M.A., Ali, A.T. and Ali, Y.M. (2017) Synthesis and Antimicrobial Evaluation of Some Novel Dithiolane, Thiophene, Coumarin, and 2-Pyridone Derivatives. Synthetic Communications, 47, 1591-1600. https://doi.org/10.1080/00397911.2017.1336776

[10] Zhou, Y., Wang, J., Gu, Z., Wang, S., Zhu, W., Acena, J.L., Soloshonok, V.A., Izawa, K. and Liu, H. (2016) Next Generation of Fluorine-Containing Pharmaceuticals, Compounds Currently in Phase II-III Clinical Trials of Major Pharmaceutical 
Companies: New Structural Trends and Therapeutic Areas. Chemical Reviews, 116, 422-518. https://doi.org/10.1021/acs.chemrev.5b00392

[11] Fujiwara, T. and O’Hagan, D. (2014) Herbicidal and Fungistatic Properties of Fluorine Analogs of Phenoxyacetic Herbicides. Journal of Fluorine Chemistry, 167, 16-29. https://doi.org/10.1016/j.jfluchem.2014.06.014

[12] Hiyama, T. and Yamamoto, H. (2000) Biologically Active Organofluorine Compounds. Springer-Verlag, Berlin Heidelberg, 137-182. https://doi.org/10.1007/978-3-662-04164-2_5

[13] Tse, B. (2013) A Versatile Method to Prepare Difluorinated Primary Alcohols and Its Application to the Syntheses of Novel Acyclic Fluorinated Nucleosides. Tetrahedron Letters, 54, 6909-6911 https://doi.org/10.1016/j.tetlet.2013.10.037

[14] Kibou, Z., Cheikh, N., Villemin, D., Choukchou-Braham, N., Mostefa-Kara, B. and Benabdallah, M. (2011) A Simple and Efficient Procedure for a 2-Pyridones Synthesis under Solvent-Free Conditions. International Journal of Organic Chemistry, 1, 242-249. https://doi.org/10.4236/ijoc.2011.14035

[15] Andrzejawska, M., Kanninski, J. and Kazimierczuk, Z. (2002) Microwave Induced Synthesis of Ribonucleosides on Solid Support. Nucleosides Nucleotides Nucleic Acids, 21, 73-78. https://doi.org/10.1081/ncn-120006532

[16] El-Sayed, H.A., Moustafa, A.H., Haikal, A.Z., Abdou, I.M. and El Ashry, E.S.H. (2008) Synthesis and Evaluation of Antimicrobial Activity of Some Pyrimidine Glycosides. Nucleosides Nucleotides Nucleic Acids, 27, 1061-1071. https://doi.org/10.1080/15257770802271805

[17] Elgemeie, G.E.H., Attia, A.M.E. and Fathy, N.M. (1994) Glycosides of Heterocycles: A Direct Route to 1-( $\beta$-D-glycopyranosyl)pyridinethione Nucleosides. Liebigs Annalen der Chemie, 955-958. https://doi.org/10.1002/jlac.199419940919

[18] Attia, A.M.E. and Elgemeie, G.E.H. (1995) Glycosides of Heterocyles. Nucleosides Nucleotides Nucleic Acids, 4, 1211-1218. https://doi.org/10.1080/15257779508010684

[19] Bauer, A.W., Kirby, W.M., Sherris, C. and Turck, M. (1966) Antibiotic Susceptibility Testing by a Standardized Single Disk Method. American Journal of Clinical Pathology, 45, 493-496.

[20] Pfaller, M.A., Burmeister, L., Bartlett, M.A. and Rinaldi, M.G. (1988) Multicenter Evaluation of Four Methods of Yeast Inoculum Preparation. Journal of Clinical Microbiology, 26, 1437-1441.

[21] Shehab, W.S. and Mouneir, S.M. (2015) Design, Synthesis, Antimicrobial Activity and Anticancer Screening of Some New 1,3-thiazolidin-4-ones Derivatives. European Journal of Chemistry, 6, 157-162. https://doi.org/10.5155/eurjchem.6.2.157-162.1219

[22] National Committee for Clinical Laboratory Standards (1997) Performance. Vol. 41, Antimicrobial Susceptibility of Flavobacteria.

[23] National Committee for Clinical Laboratory Standards (1993) Methods for Dilution Antimicrobial Susceptibility Tests for Bacteria That Grow Aerobically. Approved Standard M7-A3. National Committee for Clinical Laboratory Standards, Villanova.

[24] National Committee for Clinical Laboratory Standards (2002) Reference Method for Broth Dilution Antifungal Susceptibility Testing of Conidium-Forming Filamentous Fungi: Proposed Standard M38-A. NCCLS, Wayne.

[25] National Committee for Clinical Laboratory Standards (2003) Method for Antifungal Disk Diffusion Susceptibility Testing of Yeast: Proposed Guideline M44-P. 
NCCLS, Wayne.

[26] Liebowitz, L.D., Ashbee, H.R., Evans, E.G.V., Chong, Y., Mallatova, N., Zaidi, M., Gibbs, D. and Global Antifungal Surveillance Group (2001) A Two Year Global Evaluation of the Susceptibility of Candida Species to Fluconazole by Disk Diffusion. Diagnostic Microbiology and Infectious Disease, 4, 27-33.

https://doi.org/10.1016/S0732-8893(01)00243-7 